\title{
Du tourisme médical à la mise en place d'un espace de soins transnational. L'exemple des patients libyens à Sfax (Tunisie)
}

\section{Betty Rouland, Mounir Jarraya et Sébastien Fleuret}

\section{(2) OpenEdition}

Édition électronique

URL : http://journals.openedition.org/rfst/651

DOI : $10.4000 /$ rfst.651

ISSN : 2492-3672

Éditeur

Espaces et SOciétés (UMR 6590)

Référence électronique

Betty Rouland, Mounir Jarraya et Sébastien Fleuret, « Du tourisme médical à la mise en place d'un espace de soins transnational. L'exemple des patients libyens à Sfax (Tunisie) », Revue francophone sur la santé et les territoires [En ligne], Tourisme, Mobilités et Santé, mis en ligne le 25 octobre 2016, consulté le 06 avril 2021. URL : http://journals.openedition.org/rfst/651 ; DOI : https://doi.org/ $10.4000 /$ rfst.651

Ce document a été généré automatiquement le 6 avril 2021.

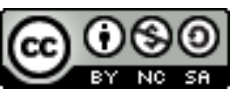

La Revue francophone sur la santé et les territoires est mise à disposition selon les termes de la Licence Creative Commons Attribution - Pas d'Utilisation Commerciale - Partage dans les Mêmes Conditions 4.0 International. 


\title{
Du tourisme médical à la mise en place d'un espace de soins transnational. L'exemple des patients libyens à Sfax (Tunisie)
}

\author{
Betty Rouland, Mounir Jarraya et Sébastien Fleuret
}

\section{Introduction}

« Le "tourisme médical", que l'on appelle également tourisme de santé ou tourisme hospitalier, se réfère au déplacement de personnes allant dans un pays autre que leur pays de résidence, dans le but de bénéficier d'un acte médical non disponible ou difficilement accessible dans leur propre pays, soit pour des raisons de législation soit pour des raisons relatives à l'offre de soins (compétences, coût) » (Hottois \& Missa, 2001).

1 Le tourisme médical est un phénomène dont l'ampleur n'a cessé de croître au cours de ces deux dernières décennies. Si les chercheurs s'accordent sur la définition d'Hottois et Missa citée en préambule, de nombreuses questions demeurent sur l'appellation même de "tourisme médical ». V. Chasles (2011) indique à ce titre que le terme est impropre dans la mesure où la motivation première du déplacement ne relève pas des motivations touristiques habituelles (loisirs, repos, récréation) mais avant tout de raisons sanitaires. Ehrbeck (2008) privilégie quant à lui le terme de voyageurs médicaux, une terminologie également utilisée par les anglo-saxons ${ }^{1}$. Du fait des imprécisions soulevées par le terme, le chiffrage du nombre de voyageurs médicaux s'avère également complexe et les données varient d'une étude à l'autre. Par exemple, A. Khelifa (2013) montre que les données oscillent dans une fourchette large, des voyageurs médicaux estimés entre 19 millions (Etude TRAM, 2006) et 40 millions de voyages par an (Keckley \& Eselius 2009 cité par Deloitte).

2 A l'origine, le tourisme médical s'est développé sur l'exploitation d'un différentiel international qui conduisait les clientèles provenant des sociétés du Nord à recourir à 
des soins moins onéreux et/ou plus rapides dans les pays du Sud. Néanmoins, ce modèle a profondément changé. On observe un élargissement du nombre de pays de provenance et de destination des patients, de leurs profils (besoins, ressources, réseaux, etc.) ainsi que des stratégies développées par les différents acteurs (professionnels de santé, patients, intermédiaires ${ }^{2}$ ). Autrement dit, aussi bien les profils des voyageurs médicaux que les échelles qu'ils mobilisent se sont diversifiés, l'amplitude des flux s'est récemment accrue au profit de mobilités intra-régionales "Sud-Sud». Des études récentes indiquent que ces mobilités "Sud-Sud» défient désormais le modèle traditionnel du tourisme médical « Nord-Sud» puisque $90 \%$ des patients qui se font soigner en Afrique du Sud proviennent des pays limitrophes contre $85 \%$ pour l'Inde et les trois quart des patients en Malaisie sont originaires d'Indonésie (Crush \& Chikanda pour l'Afrique du Sud, 2015; Ormond pour la Malaisie, 2013; Connell pour l'Inde, 2011). Les carences des systèmes de soins dans les pays d'origine (rareté des soins spécialisés, manque de personnel, structure, matériel, etc.) poussent ces voyageurs médicaux à se faire soigner de l'autre côté de la frontière.

Au regard de l'ampleur croissante du phénomène dans les régions de l'hémisphère Sud, cet article participe d'une part à enrichir un vaste "chantier " scientifique encore peu exploré en Afrique du nord et contribue, d'autre part, à fournir des données relatives à certains impacts de la révolution arabe dans la région. Soumise aux aléas d'un conflit armé diminuant l'accès aux soins en Libye et à l'augmentation des services de santé en Tunisie, la situation régionale s'est transformée au cours de ces dernières années. Cet article propose d'appréhender les modes de recours aux soins des patients se déplaçant pour des raisons de santé dans un contexte géopolitique spécifique : les libyens qui se font soigner dans la ville tunisienne de Sfax depuis la révolution arabe de 2011. Aussi, cette recherche porte sur une forme "hybride » de déplacement lié à la santé, à la croisée entre le recours aux soins transfrontaliers, le voyage et le tourisme médical. Basée sur un important travail de terrain, cette étude suggère que le voyage médical et ses corollaires (offre de soins, hébergements, aménités liées à l'accueil des patients et de ses proches) est à la fois : (i) un bon indicateur des impacts et des enjeux de la révolution arabe (spécialement en termes de reconfigurations socio-spatiales et d'enjeux économiques) ; (ii) révélateur de nouvelles logiques territoriales impulsées par divers acteurs (professionnels de santé, investisseurs, etc.) et (iii) un exemple empirique permettant de reconsidérer les dynamiques régionales et transnationales des voyageurs médicaux des pays de l'hémisphère sud.

\section{Les voyageurs médicaux libyens en Tunisie}

4 La présence de voyageurs médicaux libyens en Tunisie est concomitante au fait que le tourisme de santé constitue un secteur stratégique pour le développement des Etats (Menvielle et Menvielle, 2013; Menvielle, 2012) et la Tunisie semble bien placée dans les services de soins esthétiques, de bien-être et de confort (Boumedienne, 2012). Cette situation résulte à la fois des politiques mises en œuvre au lendemain de l'indépendance du pays, à la réouverture de la frontière tuniso-libyenne et la possibilité de circuler librement (Boubakri, 2015) et aux initiatives privées locales entreprises suite à la dégradation progressive du secteur de santé publique depuis les années 1990 .

5 La Tunisie dispose aujourd'hui de ressources sanitaires équivalentes à celles des pays développés (niveau de qualification des praticiens, standardisation des protocoles 
thérapeutiques, infrastructures sanitaires, matériel médical, etc.) à des prix très concurrentiels sur le marché mondial (Lautier, 2013). En dépit de la proximité géographique avec l'Union Européenne (UE), ce sont les patients originaires des pays voisins de la rive sud de la Méditerranée qui ont majoritairement répondu à l'offre médicale privée qui s'est développée en Tunisie. La Libye et depuis peu l'Algérie sont ainsi devenus des foyers émetteurs de patients qui passent la frontière pour se soigner et qui, contrairement aux voyageurs médicaux venus du Nord, recourent à une gamme de soins beaucoup plus large. Néanmoins, les motivations poussant les libyens à venir se faire soigner en Tunisie (ou en Jordanie) avant la révolution reposaient non pas sur l'absence de ressources sanitaires plus abondantes en Libye qu'en Tunisie mais bel et bien sur des différences de qualité de services (Gobrecht et al . 2004) ${ }^{3}$. Paradoxalement, si les services de santé tunisiens attirent une patientèle originaire des pays voisins, la population autochtone a un accès limité à l'offre privée ${ }^{4}$, faute de ressources suffisantes pour faire face au coût de ces services de santé.

6 La Tunisie se positionne actuellement comme le principal pays exportateur de soins en Afrique du Nord, le pays affiche même l'une des plus fortes croissances au monde dans le secteur du tourisme médical (Lautier, 2013) .

7 Mainvielle (2012) estime à plus de 40000 le nombre de patients étrangers accueillis chaque année en Tunisie (dont $80 \%$ de Libyens) et chiffre à 22.1 millions de Dollars les exportations de services de santé de la Tunisie (dont 18 millions USD vers la seule Libye) tout en précisant qu'il convient d'ajouter à ces chiffres les dépenses relatives aux séjours des malades (accompagnants, apport de devises, etc.). Cependant, les impacts de ce secteur d'activité tout comme les effectifs réels des libyens qui viennent se faire soigner en Tunisie échappent aux instruments de mesure: "ce phénomène n'en est probablement qu'à ses débuts et son ampleur réelle apparaît incertaine. Il est difficile à quantifier [...]. Une première difficulté de mesure est liée à la prise en compte de ces flux car ils se soumettent mal aux instruments de mesure disponibles » (Lautier, 2013).

Depuis le printemps arabe, la scène géopolitique s'est métamorphosée en Afrique du Nord entraînant de profondes mutations sur les paysages politiques, socioéconomiques, migratoires et sanitaires de part et d'autre de la frontière tunisolibyenne. Tandis que les deux gouvernements auto-proclamés en Libye (le gouvernement de Tobrouk et le Congrès national général de Tripoli) peinent à s'accorder créant des brèches qui profitent à l'implantation des groupes islamistes, la Tunisie amorce une transition démocratique encourageante (Rouland \& Bachmann, 2015). L'instabilité sécuritaire de la région mobilisée par une frontière à " haute tension » entre les deux pays n'a pourtant guère stoppé la circulation des voyageurs médicaux libyens, bien au contraire. L'éclatement de la guerre civile en Libye a fortement diminué l'offre sanitaire dans le pays et, par conséquent, a bouleversé les tendances de ce marché. Les libyens qui fréquentaient déjà la Tunisie continuent à s'y rendre en nombre pour se faire soigner redistribuant la donne en termes d'offre de soins et dynamisant plus globalement l'ensemble des activités corollaires liées au secteur du tourisme médical (hébergement, transport, alimentation, etc.).

9 Au lendemain de l'embargo de l'ONU à l'encontre de la Libye en 1992, la Tunisie est devenue une destination de premier choix pour les libyens qui choisissaient déjà de s'y faire soigner et cela s'explique par plusieurs facteurs: coût, compétences, services, proximité géographique, facilité linguistique, etc. Une situation qui s'est fondée sur des logiques de voisinage et facilitée dès 1988 par l'ouverture de la frontière tuniso- 
libyenne : libre circulation des personnes et suppression des visas (Boubakri, 2015). Dès lors, un sous-système spatial original a émergé entre la Tunisie et la Libye reposant sur un processus de complémentarité qui se lit entre l'offre et la demande ainsi que sur la qualité et le coût des soins. La patientèle libyenne représente la principale rente du tourisme médical du pays depuis l'essor du secteur (Lautier, 2005).

10 Parallèlement, le système de santé public tunisien se dégrade de façon continue depuis les années 1990. Ces deux phénomènes concomitants ont conduit à l'augmentation des investissements privés impulsés par des initiatives issues principalement des professionnels de santé. Le nombre des établissements sanitaires ainsi que celui du personnel (para)médical travaillant pour le secteur privé (qui accueille les touristes médicaux contrairement au secteur public) a ainsi considérablement augmenté (Cf. Tableau 1). Entre 1990 et 2008 , le nombre de cliniques (ou polycliniques) ${ }^{6}$ a triplé (passant de 33 à 99) sur le territoire tunisien, le nombre de centres de dialyse a quasiment sextuplé (de 18 à 105), le nombre d'officines de laboratoire a presque doublé (de 1055 à 1808) (Achour, 2011).

Tableau 1 : Augmentation du personnel de santé en Tunisie entre 2000 et 2014

\begin{tabular}{|lccccccccccccccc|}
\hline Personnel & 2000 & 2001 & 2002 & 2003 & 2004 & 2005 & 2006 & 2007 & 2008 & 2009 & 2010 & 2011 & 2012 & 2013 & 2014 \\
\hline $\begin{array}{l}\text { Nombre total } \\
\text { de médecins }\end{array}$ & 7444 & 7767 & 7964 & 8189 & 9805 & 9422 & 9653 & 10086 & 11533 & $\ldots$ & 12996 & 13686 & 12958 & 14107 & 14127 \\
\hline $\begin{array}{l}\text { Nombre de } \\
\text { médecins de } \\
\text { santé } \\
\text { publique }\end{array}$ & 4147 & 4327 & 4440 & 4522 & 5434 & 4727 & 4778 & 4818 & 5801 & $\ldots$ & 6723 & 6971 & 6058 & 6988 & 6844 \\
$\begin{array}{l}\text { Nombre de } \\
\text { médecins de } \\
\text { libres } \\
\text { pratiques }\end{array}$ & 3297 & 3440 & 3524 & 3667 & 4371 & 4695 & 4875 & 5268 & 5732 &. & 6273 & 6715 & 6900 & 7119 & 7283 \\
\hline $\begin{array}{l}\text { Nombre total } \\
\text { du personnel } \\
\text { paramédical }\end{array}$ & 27392 & 27127 & 28629 & 29030 & 29584 & 29607 & 30812 & 31312 & 32270 & 32271 & 34195 & 38077 & 28656 & 39358 & 43197 \\
\hline $\begin{array}{l}\text { Nombre de } \\
\text { techniciens } \\
\text { supérieurs }\end{array}$ & 7500 & 7284 & 7984 & 8356 & 8549 & 8677 & 8910 & 9176 & 9611 & 9611 & 10359 & 11411 & 9798 & 12160 & 13346 \\
\hline $\begin{array}{l}\text { Nombre } \\
\text { d'infirmiers }\end{array}$ & 19814 & 19843 & 20645 & 20674 & 21035 & 20930 & 22328 & 22136 & 22659 & 22660 & 23836 & 26666 & 18858 & 27198 & 29851 \\
\hline
\end{tabular}

Source : Ministère de la Santé, Institut National de la Statistique, 2016

\section{Spécificité du terrain d'étude sfaxien : de l'offre publique au secteur privé}

11 En polarisant la quasi majorité de la clientèle libyenne (accueillant respectivement $48,6 \%$ et $46,6 \%$ du total des patients libyens en Tunisie) (Lautier, 2005), les villes de Tunis et de Sfax se sont rapidement spécialisées dans les services de santé. Chef-lieu du gouvernorat qui porte son nom et seconde ville du pays en nombre d'habitants (approximativement 600000 habitants en 2014), la ville de Sfax fait figure de "porte » du sud tunisien (Cf. Figure 1). 
Figure 1 : Carte de la localisation de la ville de Sfax

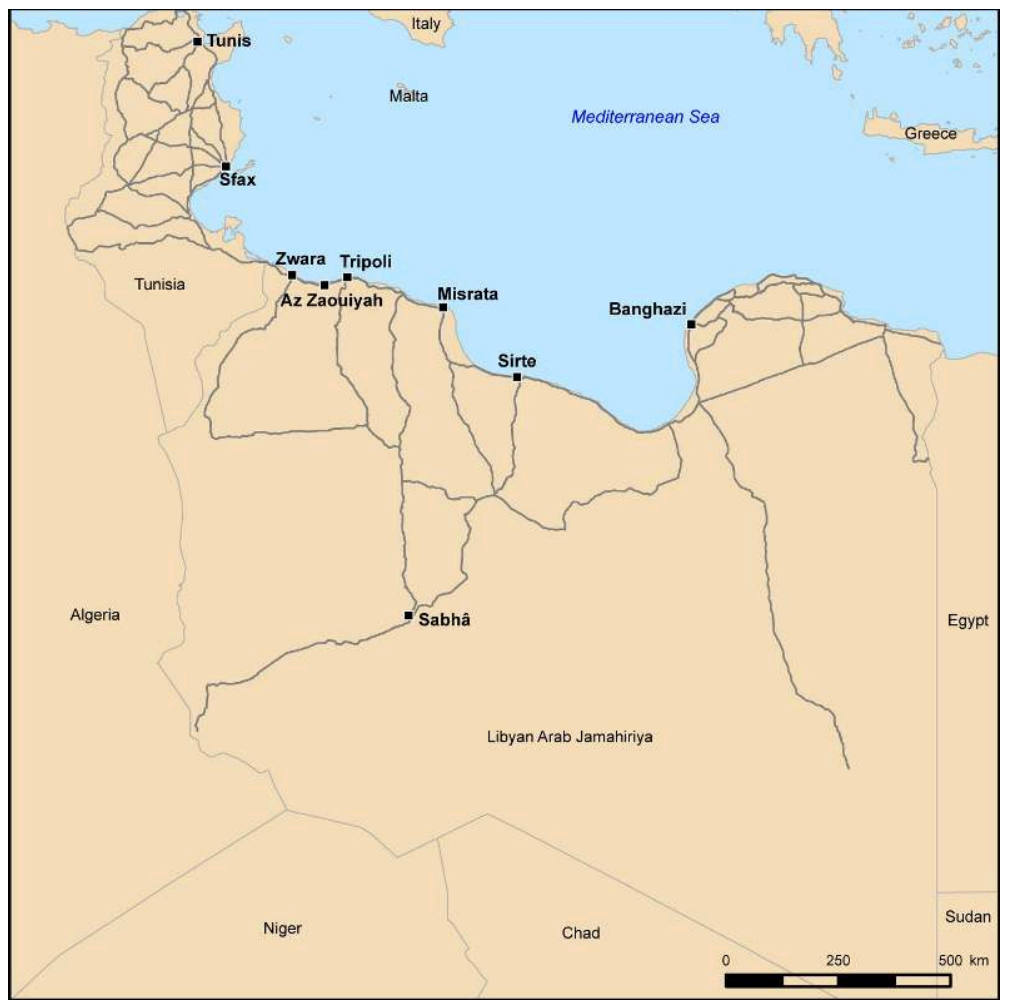

Située sur la frange littorale, l'absence d'aménagements touristiques balnéaires a contribué à alimenter une mauvaise image de Sfax perçue comme un espace urbain de production «industrielle» (activité portuaire, agricole, phosphate, etc.). Un espace considéré donc comme peu attrayant et peu récréatif qui se caractérise par une offre hôtelière limitée. A l'inverse des autres gouvernorats côtiers où l'offre balnéaire s'est largement développée, la ville de Sfax dispose de certains atouts qu'elle a très tôt su saisir pour se spécialiser dans une niche d'activité originale, à savoir les services de santé. La ville concentre localement une densité élevée de ressources sanitaires publiques qui s'avère profitable au développement de l'offre privée de services de santé (Cf. Figure 2). Outre le Centre Hospitalo-Universitaire (CHU) Hédi Chaker construit à l'époque coloniale (1927), Sfax est dotée d'une Faculté de Médecine (construite en 1974) ainsi que d'un deuxième CHU " Habib Bourguiba » (érigé en 1985). À cela s'ajoute l'implantation de structures intermédiaires dans les délégations limitrophes (3 hôpitaux régionaux), dans l'agglomération (2 Centres Intermédiaires) ainsi qu'un renforcement continu du réseau des Centres de Santé de Base $(\mathrm{CSB})^{7}$. L'installation des deux CHU dans le centre-ville de Sfax explique la polarisation continue des flux de patients originaires des gouvernorats du Sud et du Centre-Ouest du pays, inégalement desservis par les services de santé publique (Jarraya et al ., 2007; Jarraya et Beltrando, 2013). Les ressources sanitaires publiques locales ont ainsi fortement contribué à l'émergence du secteur privé. Bénéficiant d'un corps médical formé, la fonction universitaire des hôpitaux de Sfax a permis de développer, d'alimenter et de consolider de façon continue les besoins du secteur privé dans l'agglomération. Une situation favorisée par la détérioration des structures hospitalo-universitaires qui souffrent, faute de moyens, de multiples problèmes (congestion des services, dégradation des équipements, insuffisance de matériel et de personnel, etc. $)^{8}$. Face à cette situation, la construction d'un nouveau CHU a été programmée depuis 2009 mais les travaux tardent 
de commencer ${ }^{9}$. Les services de santé privés sont concentrés dans l'agglomération et comprennent: des cabinets médicaux de "libre pratique " (998 médecins de libre pratique dont 563 spécialistes en 2012), des centres d'explorations radiographiques et biologiques ( 32 centres de radiologie et 45 laboratoires d'analyses biologiques), des polycliniques (14 polycliniques dont deux en construction), etc. (Ministère de la Santé Publique, 2012).

À la veille du printemps arabe, des liaisons aériennes hebdomadaires entre Sfax et les villes libyennes de Tripoli, Misrata et Benghazi assuraient le transport des patients. En 2009, une ligne maritime reliant Sfax-Tripoli avait été créée pour un total de 1400 passagers et 400 voitures. L'accès à la ville a toutefois considérablement diminué depuis la révolution de 2011, les lignes aériennes et maritimes sont fermées (difficultés financières, sécuritaires et techniques). Le transport routier reste désormais le principal moyen de transport utilisé via la route qui longe le littoral et passe par les gouvernorats du sud-est tunisien (Médenine et Gabès) rejoignant la ville de Sfax.

Figure 2 : Répartition du nombre de médecins des secteurs public et privé de santé dans les gouvernorats tunisiens en 2011

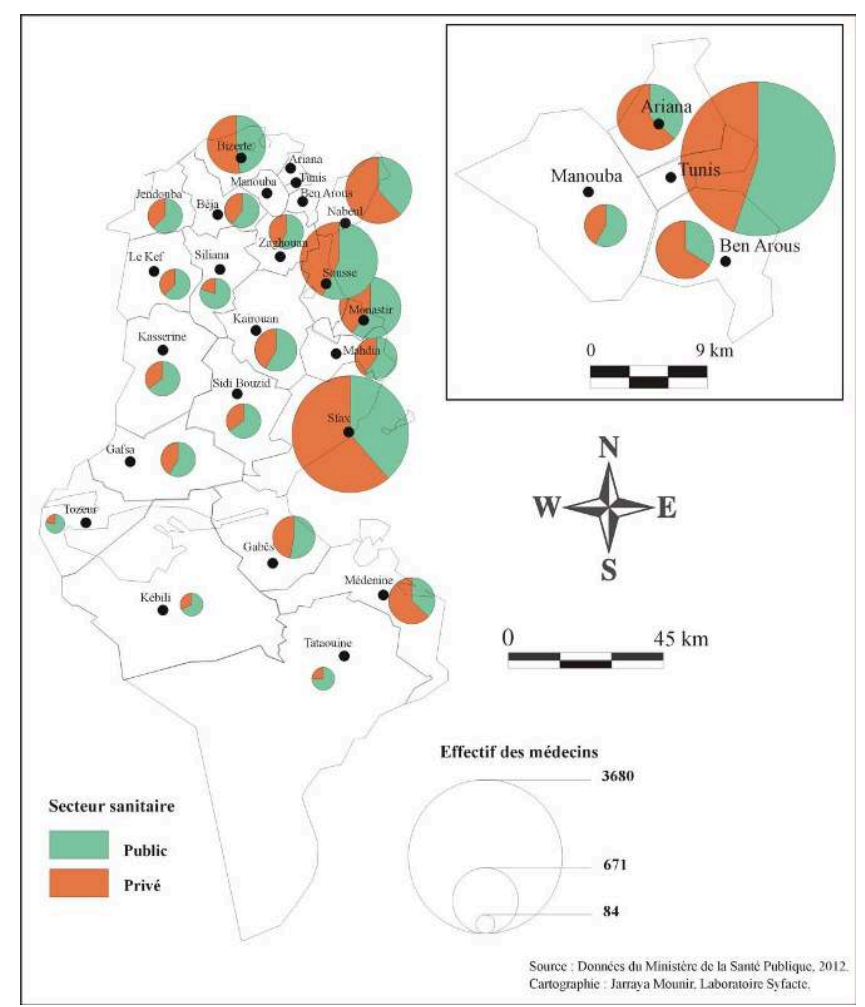

\section{Une méthodologie hybride adaptée à un terrain difficile}

14 Afin de combler un manque généralisé de données sur le sujet proposé, notre démarche s'appuie sur une méthodologie exploratoire et un exemple localisé visant à obtenir des informations de "première main ». À partir d'une série d'entretiens menée auprès des professionnels de santé et d'une enquête par questionnaire conduite auprès des patients libyens dans quatre polycliniques privées de la ville de Sfax, cette étude analyse conjointement les logiques des services sanitaires privés sfaxiens et les modes de recours aux soins des patients libyens. Une attention particulière est portée à 
l'examen des stratégies développées par les différents acteurs avant et après la révolution arabe de 2011 ainsi qu'aux logiques territoriales déployées.

Les entretiens semi-directifs ont porté sur les évolutions observées par les professionnels de santé vis-à-vis de(s) mode(s) de recours aux soins de la patientèle libyenne en Tunisie, plus spécialement les transformations enregistrées à la suite du printemps arabe. Pour les professionnels travaillant dans une polyclinique de la ville, une série de questions concernait spécifiquement la structure (organisation, financement, fonctionnement, services, partenariats, etc.) et leurs relations avec la patientèle libyenne (proportion de la clientèle libyenne, profil et besoins de ces patients, mode(s) de prise de contact et de recours aux soins, etc.). Au final, nous avons interrogé quatre médecins spécialistes travaillant dans le secteur privé (ORL, cardiologue, pédiatre, chirurgien orthopédique), le surveillant général d'une polyclinique et un médecin chef de service d'une unité de soins dans un hôpital public (également professeur à la faculté de médecine). Des entretiens auprès du personnel " extra-médical » ont également été conduits en interrogeant le directeur financier d'une polyclinique (également à la tête d'une société de tourisme médical ${ }^{10}$ ) et nous nous sommes aussi entretenus de manière moins formelle avec des membres d'associations menant des activités auprès des libyens résidant en Tunisie (Médecins du monde, Organisation Internationale des Migrations, etc.).

D'un autre côté, l'enquête par questionnaire réalisée de la mi-mai à la mi-juin 2015 auprès des patients libyens $(\mathrm{n}=205)$ s'articule autour de quatre axes visant à: contextualiser la situation familiale et socio-économique de l'individu (état civil, pays de scolarisation des enfants, catégorie socio-professionnelle, etc.) ; identifier le type de mobilité (motif principal et durée du séjour, fréquence des séjours médicaux, itinéraire, réseau, etc.); examiner l'état de santé ainsi que le(s) mode(s) de recours aux services sanitaires (état de santé physique et psychologique déclaré par la personne interrogée, opinion vis-à-vis de la structure et du personnel soignant rencontré); évaluer les conditions du séjour à Sfax (logement, prise en charge, etc.). Dans la mesure où peu de statistiques fiables existent sur le nombre de libyens qui résident ou séjournent en Tunisie, une enquête « spatialisée » a été privilégiée. Echantillonner le questionnaire en fonction de données officielles et/ou de variables compositionnelles s'avère un exercice délicat (voire impossible), on a donc opté pour une entrée de terrain en passant directement par les structures de soins. L'enquête s'est réalisée dans quatre polycliniques construites à des dates variables et situées sur des axes routiers stratégiques de la ville (cf. Tableau 2). Ce choix a été motivé par le fait que les conditions d'enquête de terrain facilitaient l'accès aux patients libyens autour des structures. Néanmoins, nous avons recueilli un faible nombre de questionnaires à Chams car, la polyclinique était en cours de rénovation pendant l'enquête et peu de patients libyens étaient présents. 
Tableau 2 : Nombre de questionnaires réalisés en fonction de la polyclinique enquêtée (Résultats de l'enquête " Migration libyenne et essor du secteur privé de la santé dans la ville de Sfax »,2015)

\begin{tabular}{|lllll|}
\hline & $\begin{array}{l}\text { Date } \\
\text { construction }\end{array}$ & $\begin{array}{l}\text { de } \\
\text { géographique }\end{array}$ & $\begin{array}{l}\text { Libyens } \\
\text { interrogés }\end{array}$ & $\begin{array}{l}\text { Fréquence } \\
\text { réponses }\end{array}$
\end{tabular}

La composition finale de l'échantillon de population enquêtée témoigne d'un fort déséquilibre: plus des deux tiers des interrogés sont des jeunes hommes actifs $(66,3 \%$ de 20-44 ans contre $24,4 \%$ entre $45-64$ ans) alors que le nombre total de femmes rencontrées reste relativement faible $(5,9 \%)$. Cet écart est lié à une réalité de terrain particulièrement difficile partagée par l'ensemble des professionnels travaillant auprès de la population libyenne en Tunisie. Dans la mesure où les femmes libyennes fréquentent moins (voire pas du tout) les espaces publics, elles sont beaucoup plus difficiles d'accès ${ }^{11}$. Les médecins interrogés nous ont signalé que le nombre de femmes libyennes qui consultent ou accompagnent les patients a diminué au cours de ces derniers mois en raison de l'augmentation de l'insécurité pour rejoindre la frontière tuniso-libyenne. Les résultats soulignent néanmoins que les trois quart des individus interrogés viennent à Sfax «avec la famille» $(77,6 \%)$ tandis que plus des deux tiers répondent être marié(e)s et avoir des enfants ${ }^{12}(69,8 \%)$.

\section{Le développement des services de santé sfaxiens, initiatives locales et marqueurs de dynamisme économique dans l'espace urbain}

18 En nous basant sur les récits collectés auprès des professionnels de santé interrogés ainsi que sur la littérature scientifique existante, le développement du tourisme médical dans la ville de Sfax paraît suivre quatre phases d'évolutions distinctes: le succès des premières polycliniques auprès de la clientèle libyenne dans les années quatre-vingt et quatre-vingt-dix; l'accélération des investissements privés et l'ancrage de la niche d'activité dans les années 2000 ; la révolution arabe et la crise du secteur en 2011 ; le contexte géopolitique régional et le dynamisme " post-révolution ».

19 Tout d'abord, les praticiens soulignaient systématiquement pendant les entretiens l'analogie entre le déclin du système de santé tunisien pendant le règne de Ben Ali et l'émergence des services sanitaires privés. Aussi, ils évoquaient une liste de problèmes structurels récurrents pour justifier l'essor du secteur privé de santé, une niche d'activité qu'ils qualifient de "refuge » en mentionnant entre autres: une prise en charge de la Caisse Nationale d'Assurance Maladie tunisienne (CNAM) inadéquate aussi bien pour les patients que pour les praticiens ${ }^{13}$; une dégradation généralisée du système de soins (insuffisance du nombre de lits d'hôpitaux, vétusté des structures 
exposant à des risques d'infections nosocomiales, délai d'attente trop long pour un acte chirurgical) ou le blocage du matériel médical et la corruption à la douane; des salaires peu attrayants ${ }^{14}$. Ce glissement progressif des médecins vers le secteur privé a été possible grâce à la mise en place de dynamiques territoriales innovantes qui combinent des initiatives locales impulsées par les praticiens sfaxiens et des formes de circulations à l'échelle de la sous-région.

20 Les premières polycliniques privées ouvertes à Sfax se sont implantées dans le centreville ${ }^{15}$ ou à proximité à la fin des années quatre-vingt, une offre spécialisée à laquelle la clientèle libyenne a très rapidement répondu. Au-delà des effets de contiguïté géographique avec une ville qui fait office de "porte » du sud tunisien, la polarisation de la clientèle libyenne à Sfax a été facilité par l'existence de réseaux migratoires libyens anciennement implantés (tourisme, affaire, mariage) :

"A Sfax, il y a une histoire ancienne avec les libyens, des échanges depuis longtemps comme les mariages; les libyens sont propriétaires de maisons: ce n'est pas nouveau. Par contre, les algériens c'est plus récent » (Surveillant général dans une polyclinique, Mai 2015 à Sfax).

21 Plusieurs facteurs étaient réunis pour favoriser l'essor de cette niche d'activité originale: embargo onusien sur la Libye poussant aux logiques de voisinage; un réservoir de main d'œuvre locale grâce au centre hospitalier universitaire ; des services de qualité à des prix très compétitifs ; etc. Une nouvelle opportunité économique était donnée à la ville restée en marge du développement du tourisme balnéaire tunisien.

22 Tout au long de la première décennie de ce siècle, l'accroissement des investissements privés a consolidé l'essor des services sanitaires notamment grâce à l'attractivité des patients Libyens et dans une moindre mesure Algériens. Observant le succès des premières polycliniques auprès de la clientèle libyenne ainsi que l'allongement des listes d'attente, les professionnels de santé ont très tôt saisi le potentiel économique du secteur d'activité devenant des piliers de l'entreprenariat local. Le nombre de polycliniques est passé de trois à douze entre 1998 et 2011 à Sfax ${ }^{16}$. Les professionnels de santé entreprirent de s'associer en investissant collectivement dans la construction de polycliniques ${ }^{17}:$ "Les cliniques privées sont des initiatives qui viennent des médecins et de la banque locale $e^{18}$ : au lieu d'acheter des appartements à Hammamet, on devient actionnaire dans une polyclinique " (Médecin ORL, actionnaire dans une polyclinique, Avril 2015 à Sfax). Les libyens représentent ce que les professionnels de santé considèrent comme un «marché porteur ». En effet, le développement des services sanitaires s'est dès le début fondé sur cette relation de réciprocité qui s'établit à la fois sur des effets de contiguiité géographique (logiques de voisinage) et des effets de discontinuités sociosanitaires (déficit de clients autochtones, qualité et coût des soins en Tunisie). Par ailleurs, la mise en place de dispositifs assurantiels a renforcé la dépendance des structures de soins sfaxiennes envers la clientèle libyenne. En effet, un système de conventions s'est développé pour faciliter la prise en charge des patients libyens sous la forme d'assurances financées soit par l'Etat Libyen, soit par des sociétés privées qui remboursent directement les polycliniques sfaxiennes. Autrement dit, aussi bien les patients que leurs systèmes de prises en charge circulent dans la région. Par conséquent, le système économique sur lequel repose les services sanitaires privés sfaxiens est dépendant du pays voisin, les libyens représentent $80 \%$ de la clientèle dans les trois quart des polycliniques sfaxiennes: 
«Dans la polyclinique Errayhane, les libyens représentent entre 80 et $85 \%$ de la clientèle, sans libyens il n'y a pas de polyclinique» (Directeur financier d'une polyclinique, Mai 2015 à Sfax).

Les médecins interrogés indiquent quant à eux que la patientèle libyenne représente $30 \%$ du total des consultations dans leurs cabinets privés.

La compréhension du développement local des services de santé ne peut pas être dissociée de l'évolution de la morphologie urbaine de la ville de Sfax, un processus de densification matérialisé par l'extension verticale non seulement du centre-ville mais aussi le long des « radiales $»^{19}$ (Cf. Figure 4). Cette forme de bâti vertical et collectif est marquée par un coût de construction qui n'a cessé de croître au cours de ces dernières années à cause d'une finition de «haut standing ". A la veille de la révolution arabe en 2011, les cabinets médicaux se concentraient dans les principales avenues du centreville (avenue du 14 janvier/avenue de Carthage) tandis que les polycliniques soumises à un processus d'étalement urbain s'implantaient stratégiquement sur les radiales. Aussi, la construction d'une polyclinique est sujette à la disponibilité des terrains :

«Dans les années 1980, Sfax était un centre-ville et rien d'autre, c'est pourquoi la clinique Bessatine s'est implantée au centre-ville» et «[...] la route El Ain, avec $6000 \mathrm{~m}^{2}$ de terrain, un choix au hasard car c'était le seul terrain disponible» (Médecins cardiologue, actionnaire dans deux polycliniques, Avril-Mai 2015 à Sfax).

Figure 4 : Concentration spatiale des polycliniques dans un rayon de 3 kilomètres de distance au centre-ville de Sfax

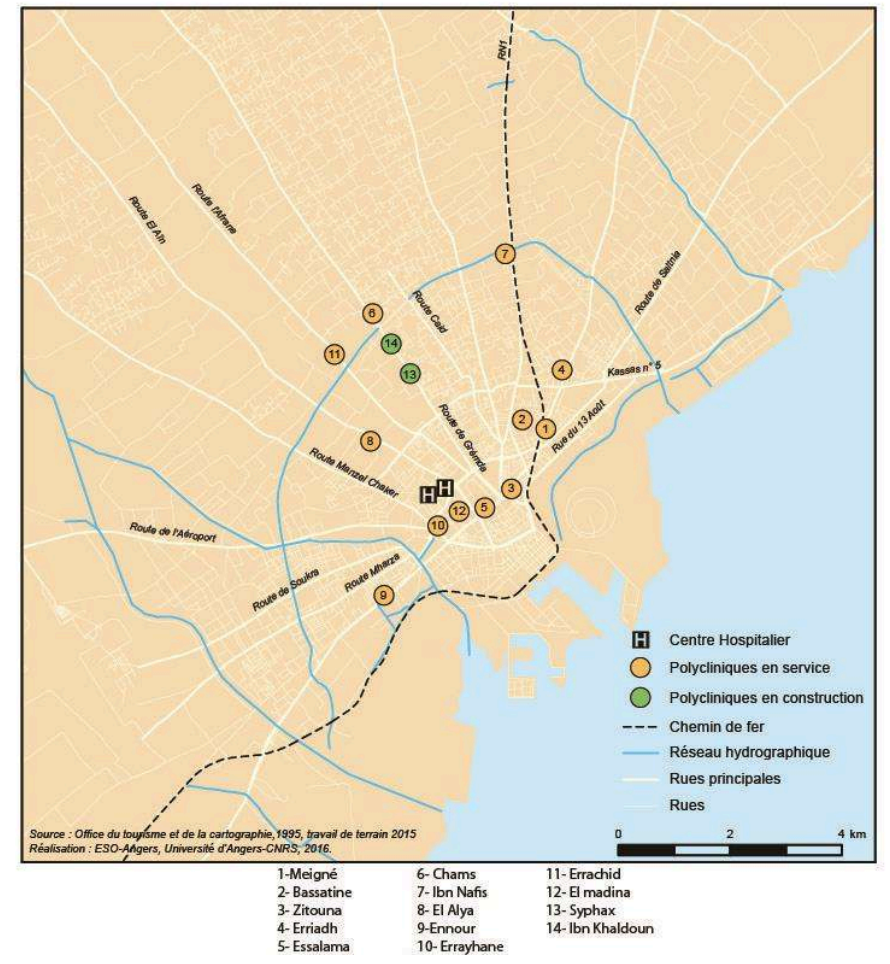

Les mouvements révolutionnaires qui se sont soulevés en Tunisie et en Libye en 2011 marquent un tournant majeur sur les dynamiques des voyages médicaux dans la région. Durant la première phase du printemps arabe, de nombreuses polycliniques ont fermé à Sfax et à Tunis stoppant brutalement la croissance du secteur en Tunisie. Or, l'éclatement des conflits en Libye a rapidement entraîné, dans une seconde phase, un 
afflux de blessés de guerre provoquant la réouverture et la congestion des polycliniques :

«En 2011, il y a eu une crise du secteur, 80 \% des patients étaient des libyens blessés de guerre, une période de situation exceptionnelle : des bateaux entiers amenaient des blessés et on avait l'obligation de les soigner » (Surveillant général dans une polyclinique, Mai 2015 à Sfax $)^{20}$.

Les polycliniques tunisiennes ont accueilli ces patients nécessitant des soins spécifiques (traumatologie, chirurgie orthopédique, etc.), des opérations très lourdes et par conséquent très coûteuses pour l'état Libyen. En parallèle à l'enlisement de la guerre civile en Libye, les factures de soins dispensés aux blessés de guerre se sont accumulées altérant les systèmes de remboursement mis en place entre les structures de soins privées sfaxiennes (et plus globalement sur l'ensemble du territoire tunisien), l'état libyen et les assurances privées.

Depuis 2012, on observe d'une part l'augmentation et la diversification des profils des patients et, d'autre part, la reconfiguration des logiques territoriales (locales, régionales, transnationales). En effet, les services sanitaires privés affichent une reprise économique bien visible sur le paysage urbain sfaxien, le nombre de patients libyens n'a cessé de croître (Cf. Figure 5).

Figure 5 : Registre du nombre de patients libyens entre 2007 et 2013 dans une polyclinique sfaxienne (source anonymisée)

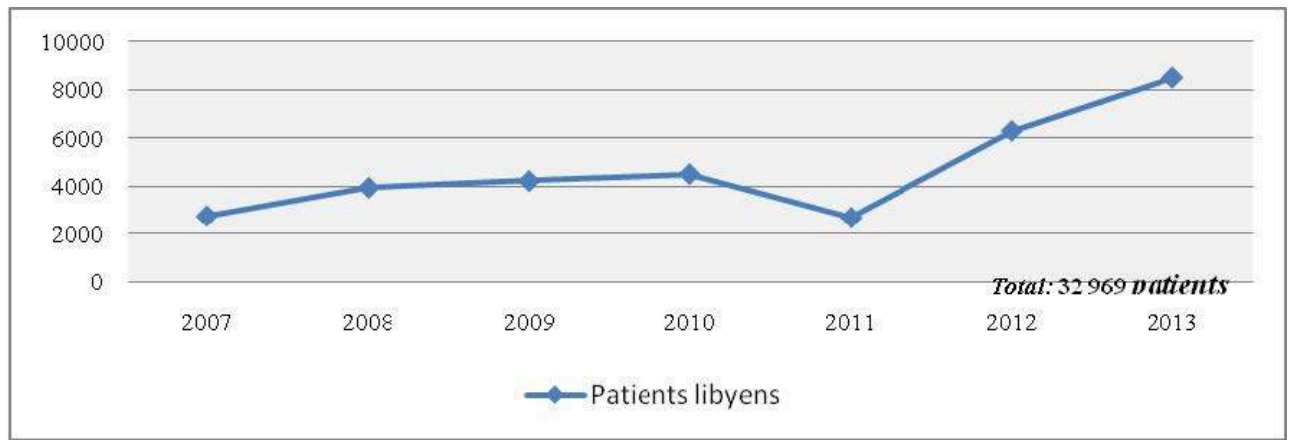

La guerre civile en Libye a non seulement entraîné la fermeture des aéroports mais le conflit a aussi diminué le nombre de services de santé dans le pays. Dès la fin de l'année 2011, le nouveau ministère de la santé Libyen demandait de l'aide à l'Organisation Mondiale de la Santé afin de reconstruire les structures de soins endommagées ou détruites dans le pays (OMS, 2016). Depuis, la situation politique en Libye ne s'est guère améliorée et la pénurie de soins n'a cessé d'augmenter, de nouveaux besoins se sont créés (ex. en santé mentale) qui s'éloignent des logiques des voyageurs médicaux libyens à Sfax avant la révolution. Située à proximité de la frontière libyenne et disposant de services de soins de qualité variés, Sfax bénéficie désormais d'une position géographique stratégique. Jusqu'alors désavantagée par les transports aériens (avant la révolution les vols étaient quotidiens pour Tunis et seulement hebdomadaires vers Sfax), l'accessibilité de la ville par voie routière facilite le transport des patients (véhicules privés, collectifs, sanitaires).

29 Le paysage urbain se métamorphose au même rythme que le nombre de patients libyens augmente, les projets d'aménagements se multiplient et on assiste aujourd'hui à une course effrénée à l'achat de terrains. Aux polycliniques en cours de rénovation s'ajoutent celles en construction ${ }^{21}$ ainsi que des nouveaux projets d'aménagements 
destinés à des activités corollaires (Cf. Photo 1 et 2). Les logiques territoriales changent et tendent désormais à concentrer l'ensemble des services (santé, commerce ou résidence). La construction d'une polyclinique s'accompagne désormais de la construction à proximité de cabinets médicaux pour diminuer le temps de trajet du praticien entre son cabinet médical et la clinique. Afin d'illustrer l'hyper concentration des structures de soins à l'échelle locale, prenons l'exemple de la route de Gremda (cf. Figure 4). Sur une distance d'un kilomètre et demi, on recense : la polyclinique Chams construite en 2006 située à $3 \mathrm{~km}$ du centre-ville (en cours de rénovation); des résidences de haut standing destinées aux patients libyens et algériens ainsi que des particuliers qui sous-louent des dépendances de leurs maisons; deux polycliniques en construction situées à quelques centaines de mètres chacune (Syphax et Ibn Khaldoun); de nouveaux aménagement à caractère commercial et para-médical (Cf. Photo 1 et 2). À titre de comparaison, on comptabilise 9 cliniques dans la ville française de Nantes dont l'agglomération est de taille comparable à Sfax (600 000 habitants environs).

Photo 1 et 2 : Aménagements commerciaux et résidentiels en construction sur la route de Gremda

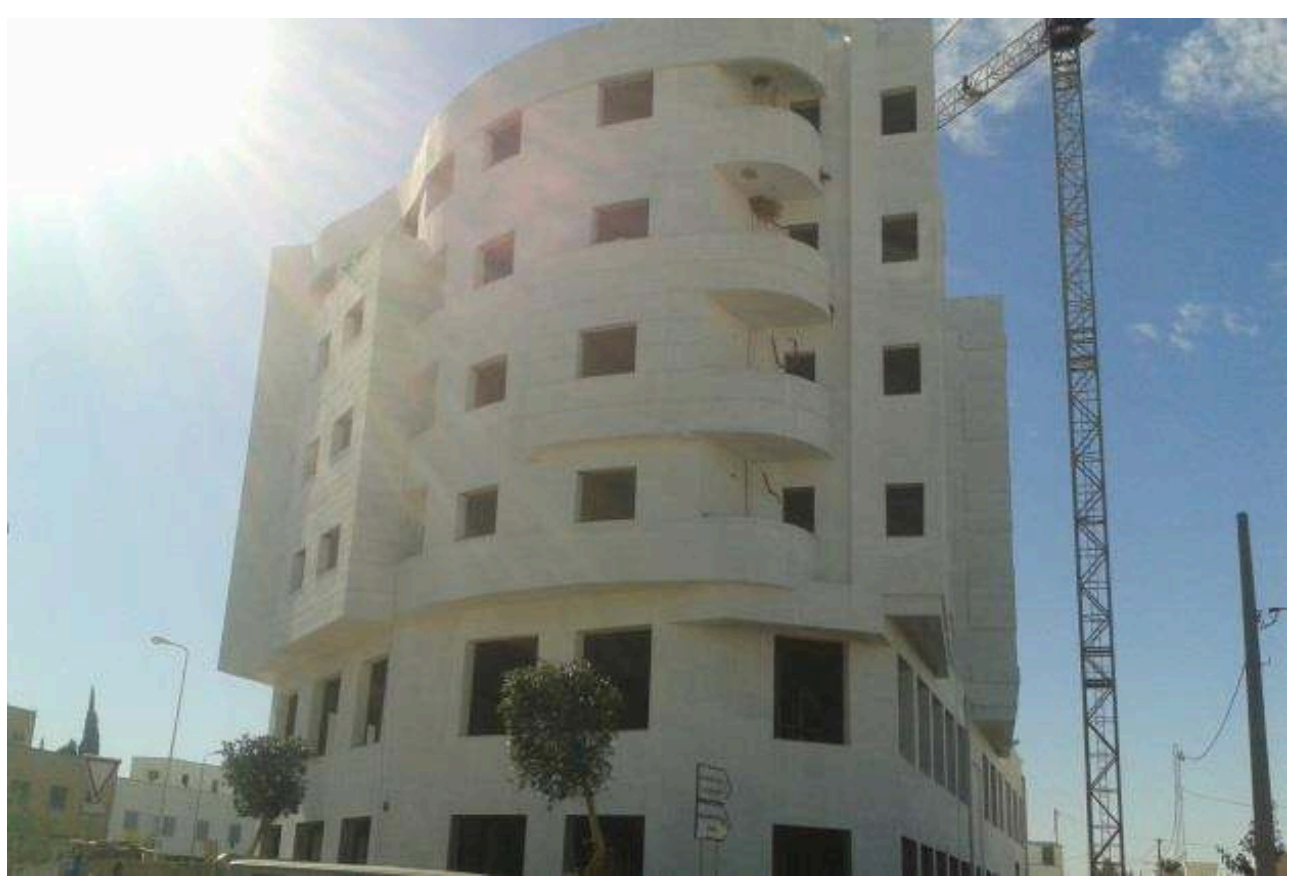

Source : Rouland Betty, janvier 2015 


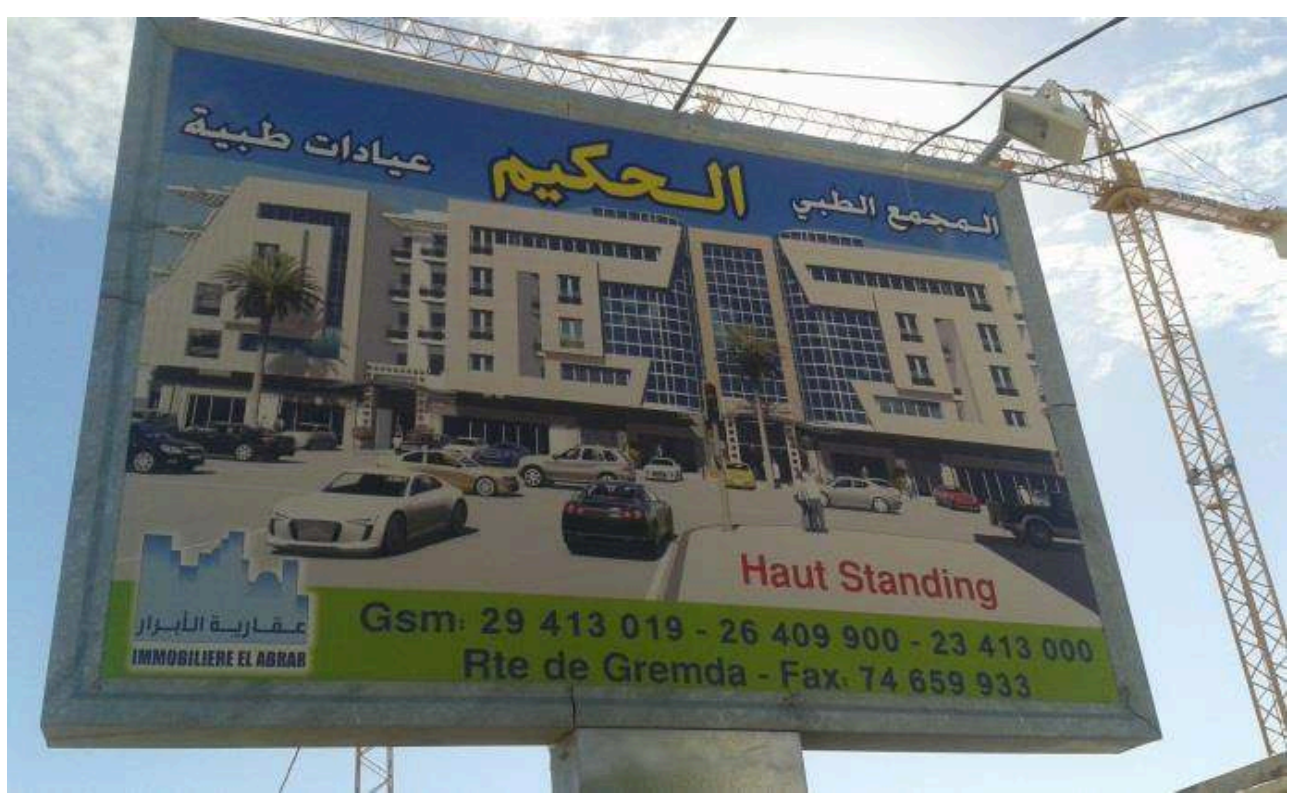

Source : Rouland Betty, janvier 2015.

\section{Vers l'émergence d'un réseau de soins transnational ?}

30 Malgré une représentativité limitée de l'échantillon enquêté de patients libyens $(n=205)$ et un contexte spécifique à la ville étudiée ${ }^{22}$, les observations collectées confirment d'abord des dynamiques migratoires caractéristiques du tourisme médical (motif, durée) fondées sur des logiques de voisinage. En effet, la grande majorité des personnes interrogées sont originaires de la région traditionnelle "tripolitaine " (91\%), c'est-à-dire des districts administratifs (Shabiyah) ${ }^{23}$ transfrontaliers ou à proximité de la frontière tunisienne dans le nord-est de la Libye (dont 37,1\% de Tripoli ; 13,2 \% d'Az Zaouiyah et $8,3 \%$ de Misrata) ${ }^{24}$. Plus des trois quart des libyens enquêtés indiquent que la santé constitue la principale raison de leur venue à Sfax ${ }^{25}$. Les résultats de l'enquête soulignent le fait que les patients interrogés valorisent les prestations dispensées dans les structures sanitaires sfaxiennes ainsi que les compétences du personnel médical local. Les variables les plus citées qui justifient le choix de se faire soigner à Sfax sont : la proximité géographique $(93,2 \%$ des observations citées), les compétences des médecins ( $55,1 \%)$, la qualité des soins $(28,3 \%)$ et les facilités linguistiques et culturelles d'un pays arabe " voisin » $(20 \%)$. Etonnamment, très peu des personnes enquêtées mentionnent la guerre civile et le manque de structures de soins, le faible coût des soins ou les facilités administratives (pas de visa) en Tunisie ${ }^{26}$. Audelà de la proximité géographique de la ville, les données recueillies soulignant la qualité des soins et la dimension culturelle font écho à un rapport publié en 2005 à propos des perspectives de développement des échanges internationaux de services de santé en Afrique du nord :

«L'exportation de services de services de santé s'inscrit en priorité dans une dimension régionale. Elle suit un «principe de voisinage ». Les pays exportateurs sont ceux qui disposent d'un avantage compétitif (rapport qualité/coût des soins) vis-à-vis de leurs voisins. Cette notion de voisinage est d'abord géographique proximité, frontière commune - mais elle peut également avoir une dimension culturelle » (Lautier, 2005). 
31 Concernant la durée des séjours, les deux tiers des personnes interrogées déclarent être à Sfax depuis une date inférieure à une semaine et quasiment le même nombre prévoit de rentrer dans un délai inférieur ou égal à deux semaines ${ }^{27}$. Autrement dit, les libyens rencontrés sont bel et bien des patients de " passage ", seulement un très faible nombre d'entre eux réside de manière permanente à $S f a x^{28}$. La fréquence des séjours sanitaires réalisés avant et après la révolution de 2011 témoigne de nouvelles vagues de patients qui empruntent l'espace de soins transnational préalablement tissé par les voyageurs médicaux libyens (Cf. Tableau 3). Parmi les 53 individus qui répondent effectuer le premier séjour de l'année à Sfax, un tiers des personnes interrogés n'était jamais venu en Tunisie avant 2011 et effectuent le premier voyage médical au moment de l'enquête.

Tableau 3 : Nombre de séjours déclarés par les libyens venants pour des raisons de santé avant et post-révolution (Résultats de l'enquête, mai-juin 2015)

\begin{tabular}{|c|c|c|}
\hline Nombre de séjours & Observations & Fréquence \\
\hline \multicolumn{3}{|c|}{ Avant 2011 en Tunisie } \\
\hline Non réponse & 1 & $0,6 \%$ \\
\hline Aucun & 37 & $20,6 \%$ \\
\hline Entre 1 et 2 & 37 & $20,6 \%$ \\
\hline Entre 3 et 5 & 44 & $24,4 \%$ \\
\hline Entre 6 et 10 & 34 & $18,9 \%$ \\
\hline$>a d 0$ & 27 & $15 \%$ \\
\hline \multicolumn{3}{|c|}{ Au cours de l'année d'enquête à Sfax } \\
\hline Non réponse & 6 & $3,3 \%$ \\
\hline ler séjour & 53 & $29,4 \%$ \\
\hline Deuxième séjour & 28 & $15,6 \%$ \\
\hline Entre 3 et 5 & 76 & $42,2 \%$ \\
\hline Entre 6 et 10 & 13 & $7,2 \%$ \\
\hline$>\dot{a} 10$ & 4 & $2,2 \%$ \\
\hline Total Observations & 180 & $100 \%$ \\
\hline
\end{tabular}

32 En dépit de l'instabilité à la frontière tuniso-libyenne, les patients libyens sont mobiles et circulent utilisant des réseaux hétérogènes (informels ou institutionnels, familiaux ou individuels, etc.) (Cf. Tableau 4$)^{29}$.

33 Des stratégies variées sont donc déployées bien que quasiment la moitié de l'échantillon (42,2\%) répond que la première personne rencontrée s'est réalisée par l'intermédiaire d'un « guide». Si l'appellation guide fait débat (certains privilégient le terme de « rabatteur » alors que d'autres préfèrent celui de " gardien ») ${ }^{30}$, ces derniers appartiennent au réseau informel jouant un rôle d'intermédiaire entre le patient, la structure de soins ou le praticien ${ }^{31}$. Les médecins insistent sur le poids du réseau familial et du système $d u$ "bouche à oreille » pour attirer la clientèle, une situation confirmée par un tiers des libyens (29,4\% des interrogés citent la variable « famille/ amis »). Enfin, un quart des libyens déclare rencontrer la première personne liée à son 
problème de santé par l'intermédiaire d'une " ambulance libyenne ". Mises à la disposition des libyens dans la ville ou acheminant directement les patients depuis la Libye, les ambulances libyennes sont bien visibles à Sfax (contrairement aux autres villes tunisiennes).

Tableau 4 : Réponse déclarée à la question « Comment avez-vous rencontré la première personne concernant votre problème de santé ? » (Résultats de l'enquête, mai-juin 2015)

\begin{tabular}{|llc|}
\hline Valeurs & $\begin{array}{l}\text { Nombre } \\
\text { d'observations }\end{array}$ & Fréquence \\
\hline Guides & 37 & $42,4 \%$ \\
Réseau d'amis/famille & 60 & $29,3 \%$ \\
Ambulance libyenne & 50 & $24,4 \%$ \\
Société d'assurance & 1 & $0,5 \%$ \\
Consulat libyen à Sfax & 2 & $1 \%$ \\
Médecin spécialisé qui l'oriente vers la polyclinique & 3 & $1 \%$ \\
"Autre" & 2 & $1,5 \%$ \\
\hline Total & 205 & $100 \%$ \\
\hline
\end{tabular}

\section{La vulnérabilité croissante des Libyens suite à la détérioration de la situation en Libye}

34 Depuis l'éclatement de la guerre civile en Libye, la vulnérabilité des patients face à l'accès aux soins et au financement du séjour médical à Sfax a considérablement augmenté. Aussi, 74,6 \% des Libyens payent grâce à des " ressources personnelles " tandis qu'un très faible nombre d'individus disposent d'une " assurance sanitaire privée » $(13,7 \%)$ ou d'une « carte d'assurance de l'état libyen » $(9,3 \%)$.

35 Nombreux sont les patients enquêtés qui rencontrent des difficultés économiques, certains ont même indiqué emprunter de l'argent à des membres de la famille (4,9\%). A l'inverse, les patients bénéficiant des aides de l'état libyen déclarent disposer d'une subvention qui oscille entre 900 et 6000 dinars (le dinar s'échange à 0.4 euros) ${ }^{32}$. Rappelons que les dépenses de santé des libyens en Tunisie étaient généralement prises en charge par les assurances (Etat libyen, sociétés privées) avant la révolution. D'ailleurs, la mise en circulation des systèmes assurantiels entre la Libye et la Tunisie a été un déterminant clé de l'essor des services privés de santé. La division politique actuelle de la Libye a remis en cause ce système assurantiel, et la diminution des ressources personnelles des libyens a conduit à l'accumulation des factures de soins impayées aux polycliniques depuis 2011. Les actionnaires des structures de soins expliquent rencontrer d'importantes difficultés pour se faire rembourser et de ce fait, certaines structures n'acceptent plus les cartes d'assurance et prennent en charge les patients à condition que les dépenses de santé soient directement payées par le patient. Les libyens les moins fortunés auraient la possibilité de se faire soigner dans les hôpitaux publics. Or, cette alternative reste non seulement peu connue mais le manque de confiance à l'égard du secteur public pourrait aussi expliquer qu'à l'exception des 
familles libyennes réfugiées à Sfax, peu des patients mobiles interrogés utilisent les structures de soins publiques ${ }^{33}$.

Concernant les conditions d'hébergement des patients et de leurs accompagnants, la ville de Sfax ne dispose pas de structures hôtelières suffisantes pour les accueillir, des stratégies variables sont donc déployées en fonction des ressources et des besoins des individus. Plus de la moitié des interrogés s'installe volontairement à proximité d'une polyclinique $^{34}$. En réponse à cette nouvelle manne économique, le marché locatif s'est diversifié et des acteurs pluriels participent à développer l'offre immobilière locale : des résidences de haut standing destiné à la patientèle libyenne et depuis peu algérienne ${ }^{35}$; des appartements situés dans les principales avenues du centre-ville à louer "à la nuitée " (48 individus précisent louer à la nuitée dans le quartier d'Ennasria); des villas autour des polycliniques et même dans certains cas des dépendances de maisons (comparables à des garages) (Cf. Photos 3). Le prix à la nuitée d'un appartement situé dans le centre-ville ou d'un logement individuel/dépendance située le long d'une radiale près d'une polyclinique oscille entre 30 à plus de 50 dinars (alors que le loyer moyen d'un appartement oscille entre 400 et 500 dinars). Si les libyens enquêtés ne souffrent pas de processus de ségrégation spatiale et se répartissent en fonction de la distribution des structures de soins, ils ont le sentiment d'être victime de discrimination au regard des prix du marché locatif. Inversement, la population locale également victime de l'augmentation du coût de la vie (locatif, denrées alimentaires, etc.) considère que cette flambée des prix est liée à l'arrivée de ces touristes médicaux perçus comme fortunés.

Photo 3 : Dépendance (à droite) et garage (à gauche) loués par des libyens à proximité d'une polyclinique à Sfax

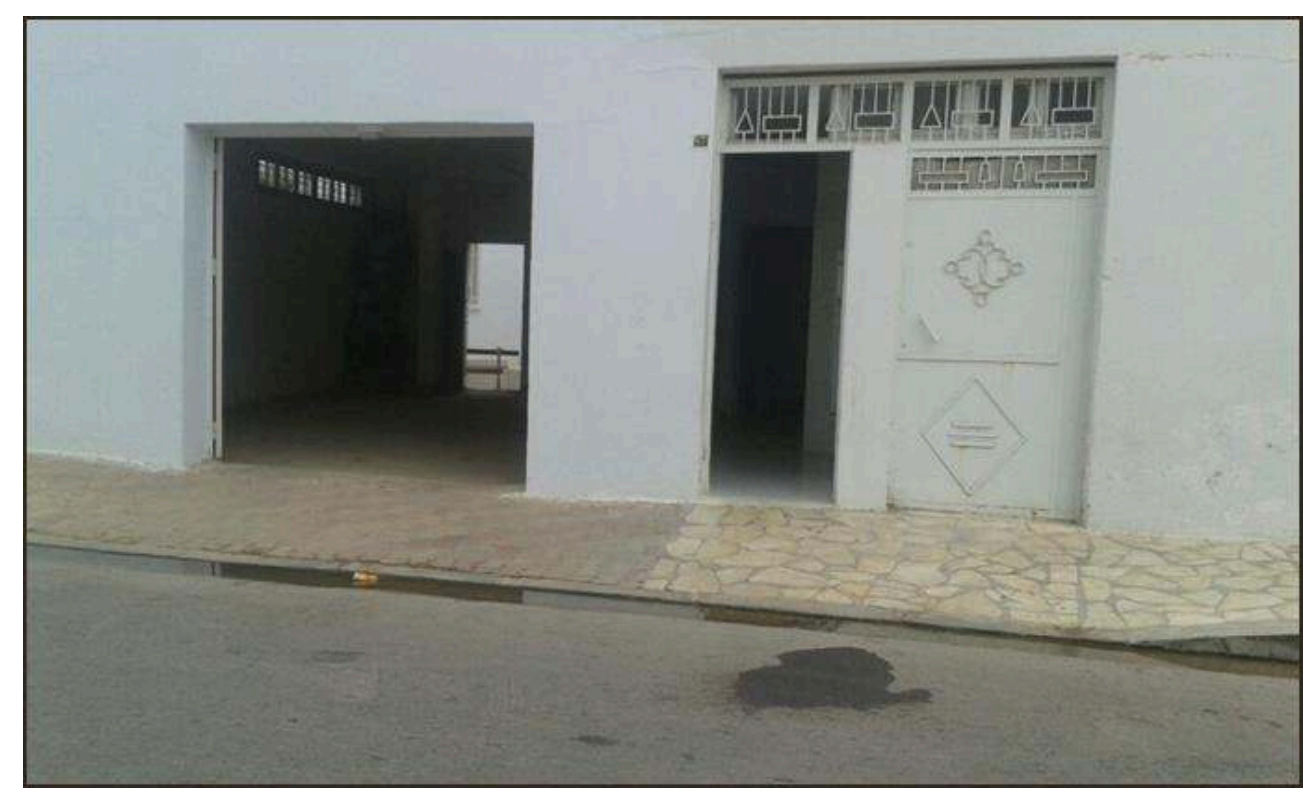

Source : Rouland Betty, 2015

37 Enfin, les paysages sanitaires qui se dégagent corroborent les informations obtenues lors des entretiens avec les professionnels de santé. Si la patientèle libyenne utilise une gamme de soins assez large, des besoins en termes de soins spécialisés apparaissent toutefois en traumatologie et en orthopédie (accidents de la route, blessés de guerre). Aussi, les médecins orthopédiques restent les médecins spécialistes consultés les plus 
cités par les enquêtés ( 39 patients), suivis des cardiologues et des neurologues ( 28 et 27 observations). La fréquence élevée des séjours médicaux des libyens à Sfax s'explique également par le besoin de soins réguliers qu'exigent certaines maladies chroniques (on a recensé 9 cas de cancer). Un quart des libyens interrogés ne donne aucune précision au sujet du médecin ou du problème de santé rencontré. Quant aux questions relatives à l'état de santé psychologique, très peu des libyens interrogé évoquent la guerre civile. Après 40 ans de régime dictatorial tenu d'une main de fer par Kadhafi, la population libyenne reste extrêmement divisée et ces questions sensibles sont donc intériorisées. Suite à l'enquête de terrain mais aussi aux entretiens menés avec les associations ${ }^{36}$, le sentiment qui domine est qu'il existe une réelle détresse psychologique difficile à appréhender par le biais d'un questionnaire.

\section{Conclusion. Du tourisme à la guerre, de la Libye à Sfax : enjeux et perspectives}

38 La crise politique libyenne a fortement participé à renouveler les flux de patients libyens à Sfax. Dotée d'une importante concentration d'infrastructures sanitaires, Sfax fait donc office de porte médicale du sud tunisien. La mise en circulation de dispositifs sanitaires spécifiques entre la Libye et les polycliniques sfaxiennes mettent en évidence l'émergence d'un espace de soins transnational . La situation géopolitique régionale a accru les besoins, les profils des patients se sont diversifiés. Sfax apparaît désormais comme une destination pragmatique plutôt qu'un choix «touristique », un espace de soins se tisse entre les deux pays grâce à la mise en circulation de dispositifs sanitaires variés (patients, systèmes assurantiels, transports ambulanciers, etc.) et la ville, développant une gamme de services corollaires, s'adapte à ces flux. Malgré le fait que l'enlisement du conflit en Libye rende incertain l'avenir du secteur, Sfax semble se spécialiser dans cette niche d'activité originale offrant localement une nouvelle opportunité économique. Les ambitions des professionnels de santé sont néanmoins bien plus importantes, la ville pourrait devenir une plateforme du tourisme médical à l'échelle de l'Afrique francophone (Afrique du nord et de l'ouest).

39 Au-delà d'offrir des données qualitatives sur un champ méconnu de la révolution arabe, cette recherche permet de reconsidérer certains enjeux de la mobilité «sudsud» des voyageurs médicaux et de s'interroger sur leurs statuts et conditions de séjours. Appauvris et vulnérables depuis l'éclatement de la guerre civile, des stratégies disparates sont déployées par les patients libyens. Difficile de les considérer comme des touristes médicaux bien qu'ils répondent à priori aux critères d'une telle définition en termes de motif et de durée de séjour. Les patients les plus vulnérables, non encadrés et empruntant des réseaux informels, correspondent à ce que Crush et Chikanda dénomme des populations « medically disenfranchised » (Crush \& Chikanda, 2015), des populations médicalement privées de leurs droits.

40 A travers la question des patients libyens qui viennent se faire soigner en Tunisie, cet article montre les limites de la catégorie englobante de "touriste médical ». A midistance entre un voyageur et un patient, dans un contexte géopolitique et historique propre à l'Afrique du Nord, les termes "touriste médical » ou "voyageur médical » apparaissent en décalage avec la réalité. N'est-il pas plutôt question ici de «mobilités médicales » ou de «mobilités pour séjours médicaux » qui obéissent à des motivations 
parfois différentes de celles qui président aux mobilités touristiques et s'inscrivent dans un contexte géopolitique régional particulier?

\section{BIBLIOGRAPHIE}

Achour, N., 2011, Le système de santé Tunisien : état des lieux et défis, UNFPA Tunisie, 24 p.

Boubakri, H. (2015). Migration et asile en Tunisie depuis 2011 : vers de nouvelles figures migratoires? ", Revue européenne des migrations internationales [En ligne], vol. $31-\mathrm{n}^{\circ} 3$ et 4 .

Boumedienne, L., 2012, Le Tourisme Médical: un Enjeu Stratégique pour la Tunisie , Institut arabe des chefs d'entreprise, 16 p. http://www.iace.tn/wp-content/uploads/2013/05/

jes2012_tourisme_medical.pdf consulté le 09/05/2016

Chasles, V., 2011. Les nouvelles dynamiques du tourisme dans le monde, Géoconfluences (February) (2011), 1-9. http://geoconfluences.enslyon.fr/doc/typespace/tourisme/ TourScient2.htm consulté le 09/05/2016

Crush, J., \& Chikanda, A. (2015). South-South medical tourism and the quest for health in Southern Africa. Social Science \& Medicine , 124 , 313-320.

Connell, J., 2011. Medical Tourism . Wallingford, Oxfordshire ; Cambridge, MA : CABI, 209 p.

EhrbeckT, and al., 2008, Mapping the market for Medical travel, Mc Kinsey Quarterly, 11 p.

Gobrecht, J. et al., 2004, Measuring Trade in Health Services, Globalization, Tradeand Health Working Paper series, juillet, OMS. https://www.imtj.com/

Jarraya M., Beltrando G., Daoud A., (2007). Congestion des structures hospitalo-universitaires à Sfax : aspects spatiaux et alternatives d'intervention. I ${ }^{\text {ème }}$ Colloque de la Géographie Maghrébine, Sfax 19-22 avril, $14 \mathrm{p}$ (Actes dactylo).

Jarraya, M., 2012, Ambiances froides et consultations respiratoires dans le secteur de la santé publique à Sfax (Tunisie), Cybergeo : European Journal of Geography . 16 p. http://

cybergeo.revues.org/25032 consulté le 09/05/2016

Jarraya, M., \& Beltrando, G., 2013. Les services de soins hospitaliers publics dans l'agglomération de Sfax: spécificités fonctionnelles et pouvoir spatial. EchoGéo , (26), 21 p.

Keckley, P. H. \& Eselius, L. L., 2009, Survey of Health Care Consumers: Key Findings, Strategic Implications. Washington: Deloitte Center for Health Solutions, $107 \mathrm{p}$.

Khelifa, A., 2013, Mondialisation des soins et tourisme médical , Etude pour l'Institut Montparnasse, 112 p. http://www.institut-montparnasse.fr/wp-content/files/Collection-recherche-n-7mondialisation-des-soins-et-tourisme-medical.pdf consulté le 09/05/2016

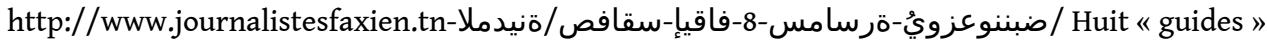
étaient arrêtés et des médecins étaient interpellés à Sfax pour « rabattage » déloyal, consulté le 10 mars 2015 


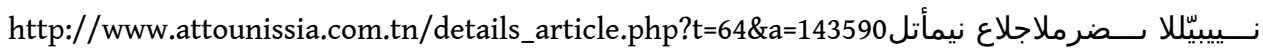
نواعت تايقافتا سنوتب Accords de coopération pour assurer l'accès aux soins des patients libyens en Tunisie, consulté le 09/05/2016

Lautier, M., 2005, Les exportations de services de santé des pays en développement : le cas tunisien . Agence française de développement, Notes et documents $n^{\circ} 25,173 \mathrm{p}$.

Lautier, M., 2013, Le développement des échanges internationaux de service de santé : perspectives des exportations en Afrique du Nord, Banque Africaine de Développement, http:// www.afdb.org/fr/news-and-events/article/international-development-of-health-services-northafricas-export-prospects-11649/

Hottois G., Missa J.N., 2001, Nouvelle encyclopédie de bioéthique , De Boeck Université, 831-832.

Menvielle L., 2012, Tourisme médical : quelle place pour les pays en développement ? Mondes en développement 1/12 ( $\mathrm{n}^{\circ}$ 157), 81-96 URL : http://www.cairn.info/revue-mondes-endeveloppement-2012-1-page-81.htm consulté le 09/05/2016

Menvielle W., Menvielle L., 2013, Tourisme médical : un secteur stratégique pour le développement des États. Revue internationale et stratégique 2/13 ( $n^{\circ}$ 90), 153-162

Ministère de la Santé Publique, 2012 : Rapport annuel des statistiques sanitaires dans le gouvernorat de Sfax. Direction Régionale de Sfax, Unité Régionale des Statistiques, 87 p.

Ormond, M., 2011. Medical tourism, medical exile: responding to the cross-border pursuit of healthcare in Malaysia. In: Minca, C., Oakes, T. (Eds.), Real Tourism: Practice, Care and Politics in Contemporary Travel Culture . Routledge, London and New York, pp. 143-161.

Rouland, B., Bachmann V., 2015,Tunisia in 2030: Perspectives and Geopolitical Challenges of a Country in Transition. The Arab World Geographer , 18 (1-2), 31-38.

\section{NOTES}

1. L' International Medical Travel Journal (IMTJ) est une plateforme d'échanges (publications, évènements, etc.) dédiée aux professionnels du secteur d'activité. Cf. https://www.imtj.com/

2. Les intermédiaires font référence ici aux agences de voyages qui se dédient au tourisme médical, aux « guides » permettant de faire la médiation entre le praticien et le patient dans le pays de destination, à certains consulats chargés des prises en charge (consulat libyen à Tunis), aux hôtels, etc.

3. On recensait une densité de 129 médecins pour 100000 habitants en Libye contre 70 en Tunisie en 2004.

4. Les données collectées sur le terrain estiment que les tunisiens représentent seulement $5 \%$ du total de la patientèle dans les cliniques privées à Sfax.

5. Basé sur des données du FMI et des estimations personnelles, Marc Lautier chiffre à plus de $20,8 \%$ la croissance annuelle des exportations des services de santé tunisiens entre 2003 et 2010, soit une croissance qui se place au 3ème rang mondial après la République Tchèque et le Brésil.

6. En Tunisie, on utilise l'appellation "polyclinique» en référence aux cliniques polyvalentes privées, des structures sanitaires offrant une large carte de services 
(capacité d'accueil comprise généralement entre une cinquantaine et une centaine de lits d'hospitalisation).

7. Au nombre de 145 CSB en 2012 (Ministère de la Santé Publique, 2012).

8. Le rallongement du délai d'attente pour une consultation constitue un bon indicateur, un rendez-vous pour un service cardiologique peut dépasser les six mois d'attente.

9. La construction de ce $\mathrm{CHU}$ est financée grâce à une donation de la Chine, le terrain a été préparé et les travaux commenceront en 2016.

10. Les sociétés de tourisme médical fonctionnent comme des agences de voyages traditionnelles sauf qu'elles se chargent de proposer des voyages médicaux incluant le coût de la prise en charge des soins et des dépenses corollaires (transports jusqu'aux infrastructures de santé, hôtels de convalescence, etc.).

11. Cette barrière à la fois socio-culturelle et politique complique le travail mené par les associations sur le terrain. Les femmes libyennes interrogées se trouvaient dans les salles d'attentes des polycliniques alors qu'on échangeait plus facilement avec les hommes dans les cafés des polycliniques, à la sortie des pharmacies, sur les parkings, etc.

12. Les résultats montrent que $77,6 \%(159)$ des individus enquêtés viennent à Sfax accompagnés de la «famille » contre $16,1 \%$ (33) «avec des ami(e)s» et 4,9\% (10) " seuls ». Concernant l'état civil, les réponses montrent que $68,8 \%$ des personnes sont mariées contre $31,2 \%$ célibataires (soit 141 individus contre 64 ), 54,1 \% déclarent avoir des enfants (113).

13. D'un côté, les consultations ne sont pas intégralement remboursées alors que le patient n'avance que $25 \%$ des frais grâce au système du tiers payant. D'un autre côté, les inégalités d'accès aux soins n'ont cessé d'augmenter au cours de ces dernières années: un couple avec deux enfants dispose d'un forfait de soins de 200 dinars/an ( 91,2 euros) soit 50 dinars par personne ( 23 euros) alors que cela correspond au prix d'une consultation chez le pédiatre. A cela s'ajoute le paradoxe des retraités qui perdent la prise en charge de leurs soins par la CNAM.

14. Le salaire d'un médecin assistant dans le public s'élève approximativement à 700 euros par mois contre 1100 euros pour un professeur agrégé en médecine et 1200 euros pour un professeur de médecine du public.

15. La polyclinique Meignié ouverte en 1962 à Sfax est la première structure de soins privée pour l'ensemble de la zones centre et sud de Tunisie suivie de l'ouverture des polycliniques Bessatine (1986) et Zaituna (1989).

16. Ouverture de la polyclinique Erriadh en 1998 ; Essalama en 2001 ; Chams, Ibn Nafis et El Alya en 2006; Ennour en 2009; Errayhane en 2010; Errachid et Sfax Jadida en 2011.

17. Par exemple, les polycliniques El Alya (2005) et Errachid (2011) sont dirigés par une quarantaine de médecins actionnaires alors que la polyclinique Bessatine regroupe des actionnaires médecins et pharmaciens. Les « polycliniques » sont des structures privées disposant d'une gamme de soins assez large.

18. La Banque africaine de développement (BAD).

19. Les radiales constituent les principaux axes routiers qui relient le centre-ville de Sfax aux municipalités voisines. Elles sont elles-mêmes connectées par trois 
« ceintures ». Certaines polycliniques portent d'ailleurs le nom de la radiale (El Alya, Errachid).

20. Une situation de crise exceptionnelle avec l'arrivée de blessés de guerre en Tunisie mais aussi dans d'autres pays à proximité comme Malte; cf. http:// www.timesofmalta.com/articles/view/20110921/local/Libyans-wounded-in-Sirte-

flown-to-Malta-for-treatment.385623 .

21. Tandis que certaines polycliniques sont en cours de rénovation (Chams) ou d'agrandissement (Errayhane), deux polycliniques sont actuellement en cours de construction et deux autres spécialisées dans les soins orthopédiques ont entrepris des démarches administratives en vue d'un projet de construction.

22. Certaines cliniques privées de la ville de Tunis rencontrent d'importantes difficultés liées à l'appauvrissement de la patientèle libyenne (cf. Boubakri, 2015, p 24).

23. Il existe actuellement 22 «Shabiyah» en Libye qui date du redécoupage administratif et territorial de 2007, le "Chabiyah» est une entité qu'on traduit par «quartier» ou «municipalité ». Ces districts équivalent à l'entité territoriale du " gouvernorat » en Tunisie.

24. A l'inverse, aucun patient libyen n'est originaire de la région du Fezzan, vaste étendue au sud de Sabhâ en Libye, et 14 libyens répondent être originaires de Benghazi.

25. Puis, $86,6 \%$ des libyens répondent affirmativement à la question «venez-vous à Sfax pour des raisons de santé ?"

26. Sur un total de 437 observations, seulement onze individus ont répondu par «le coût des soins bon marché » et sept par «facilité administrative » (pas besoin de visa) contre quatre personnes qui ont indiqué venir pour un autre motif qui n'est pas lié à la santé comme « le travail » ou la «stabilité politique et la sécurité ».

27. $17.4 \%$ des individus restent incertain indiquant qu'ils rentreront en «fonction du traitement $»$.

28. Seulement quatre individus stipulent résider à Sfax depuis plus d'un an et cinq autres indiquent que leurs enfants sont scolarisés à l'école libyenne de la ville.

29. On demandait de répondre librement à « comment avez-vous rencontré la première personne soignante, (personnel d'une structure médicale ou autre) concernant votre problème de santé ? ».

30. En arabe, le guide est appelé un "Samsar», ce qui signifie une personne intermédiaire qui assure le contact entre le patient et le prestataire de soins contre une commission d'argent payée.

31. En 2015, huit "guides » étaient arrêtés et des médecins étaient interpellés à Sfax

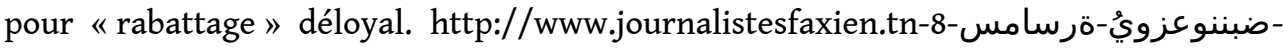
فاقيا-سقافص/ةنيدملا/ 2015 mars 10 .le

32. Un postier libyen nous a expliqué recevoir 1000 dinars d'aide pour des frais médicaux alors qu'une famille tunisienne dispose de 50 dinars pour chaque membre de la famille pour une année. Seules les personnes travaillant pour les compagnies pétrolières bénéficieraient jusqu'à l'heure actuelle du remboursement intégral des dépenses de santé.

33. Une circulaire existe indiquant à priori que les libyens ont gratuitement accès aux structures de soins publiques. L'Etat tunisien a signé un accord en décembre 2014 avec son homologue libyen qui permet d'offrir l'accès des structures hospitalières publiques 
aux patients libyens en tenant compte des moyens et de la capacité des établissements. Outre les soins ordinaires (consultations, hospitalisation et chirurgie), cet accord autorise certains centres hospitaliers tunisiens d'exécuter les actes de greffe et de transplantations. La prise en charge des soins étant assurés par l'Etat libyen.

34. Seulement quatre individus déclarent être propriétaire d'un logement à Sfax.

35. Dans la mesure où l'objet de cette recherche porte sur les patients libyens, nous avons peu de données sur la nouvelle patientèle algérienne. Cette dernière représente $15 \%$ du total des patients dans les polycliniques et le nombre devrait s'accroître dans les prochaines années selon les propos des professionnels de santé.

36. Médecins du Monde a ouvert une unité d'aide psychologique aux populations migrantes dans la ville de Tunis, les professionnels soulignent les difficultés rencontrées à échanger sur les conditions de vie et migratoires des libyens.

\section{RÉSUMÉS}

Cet article s'intéresse au triptyque "santé-tourisme-territoire » en examinant conjointement les logiques du développement des services sanitaires privés dans la ville tunisienne de Sfax et les modes de recours aux soins des patients libyens depuis la révolution arabe de 2011. Phénomène fondé sur des logiques de voisinage (réouverture de la frontière, libre circulation, suppression des visas, etc.) qui débuta dans les années 1990, le tourisme médical manifeste un fort dynamisme sur le paysage urbain sfaxien depuis 2011 et cela en dépit de l'instabilité géopolitique régionale. Sujet encore peu étudié, la méthodologie exploratoire proposée combine une enquête qualitative conduite auprès des patients libyens $(\mathrm{n}=205)$ dans quatre cliniques privées de la ville ainsi que des entretiens semi-directifs menés auprès des professionnels de santé. D'un côté, le chaos en Libye ainsi que la proximité géographique de Sfax poussent les professionnels du (para)médical à investir dans ce secteur considérant le «marché libyen » instable mais porteur. D'un autre côté, les résultats indiquent l'augmentation et la diversification des profils des patients (réseaux, ressources, besoins). Le conflit en Libye accroît en effet la vulnérabilité des populations vis-à-vis de l'accès aux soins. Les reconfigurations spatiales locales en termes d'aménagements (à caractère médical, résidentiel, commercial) et régionales en termes de circulations (patients, transports, systèmes assurantiels, etc.) expriment l'émergence d'un espace de soins transnational. Le contexte géopolitique ainsi que l'appauvrissement des patients libyens nous amène à reconsidérer le tourisme médical au profit de nouvelles formes de mobilités à caractère « médicales » et interroge la durabilité du secteur d'activité à Sfax.

This workshop concludes a cycle of 3 meetings organized in 2015 and in 2016. It relates to the problems of both the medical sector and the social sector in Romania. Entitled "Emergence and structuring of a medical and social sector in Romania", the workshop and all the former associated meetings aim at: (1) Establishing a typology of the various situations, and analysing these situations; (2) Understanding the effects of the public regulations(with social and medical dimensions) on places, their inhabitants, their establishments; (3) Analysing the reasons of the non-use of certain services; (4) Formalising the conditions of a transfer of skills and practices between France and Romania both in terms of training and of actions for the local public and/or civil. 
INDEX

Index géographique : Libye, Tunisie, Sfax

Mots-clés : tourisme de santé, espaces de soins, réseau de soins, transnational

\section{AUTEURS}

\section{BETTY ROULAND}

Chercheur Post-doctoral, Institut für Humangeographie Goethe - Universität Frankfurt/Main (Allemagne)

MOUNIR JARRAYA

Enseignant-chercheur, Laboratoire Syfacte, Université de Sfax (Tunisie)

\section{SÉBASTIEN FLEURET}

Directeur de recherche CNRS UMR ESO 